\title{
The Instrumentation Program for the Thirty Meter Telescope
}

\author{
Luc Simard*a, $^{*}$, David Crampton ${ }^{\mathrm{b}}$, Brent Ellerbroek ${ }^{\mathrm{a}}$, Corinne Boyer ${ }^{\mathrm{a}}$ \\ ${ }^{a}$ Thirty-Meter Telescope Project, 1111 South Arroyo Parkway, Pasadena, CA, USA 91105 \\ ${ }^{b}$ National Research Council of Canada, Herzberg Institute of Astrophysics, 5071 West Saanich Rd., \\ Victoria, Canada, V9E 2E7
}

\begin{abstract}
An overview of the current status of the Thirty Meter Telescope (TMT) instrumentation program is presented. Science cases and operational concepts as well as their links to the instruments are continually revisited and updated through a series of workshops and conferences. Work on the three first-light instruments (WFOS IRIS, and IRMS) has made significant progress, and many groups in TMT partner communities are developing future instrument concepts. Other instrument-related subsystems are also receiving considerable attention given their importance to the scientific end-toend performance of the Observatory. As an example, we describe aspects of the facility instrument cooling system that are crucially important to successful diffraction-limited observations on an extremely large telescope.
\end{abstract}

Keywords: Extremely large telescopes, adaptive optics, science instruments, observation planning

*Luc.Simard@nrc-cnrc.gc.ca; phone 1250 363-8315; fax 1250 363-0045; nrc-cnrc.gc.ca

\section{INTRODUCTION}

The TMT instrumentation program has made significant progress over the last two years on the instrument designs themselves as well as their end-to-end integration into the overall Observatory. This paper describes some of the milestones reached in the last two years (Section 2), progress made on the first-light instrument designs (Section 3), new future instrument concepts (Section 4) and instrument-related subsystems such as facility cooling (Section 5). The TMT adaptive optics program is described elsewhere ${ }^{1}$ in these proceedings.

\section{MILESTONES}

\subsection{Science and Instrumentation Workshop}

More than a hundred scientists and engineers converged in Victoria, British Columbia, Canada, on March 28-30, 2011, to explore new instrument and adaptive optics concepts and capabilities for TMT and their exciting scientific potential. This meeting marked an important milestone for the TMT project as scientists from all communities now forming the TMT partnership (including its newest members China and India) came together for the first time to lay down the next steps in TMT's scientific mission. Input from this meeting helped the TMT Scientific Advisory Committee (SAC) establish priorities for second-generation instrumentation capabilities. The program for the workshop featured a full menu of science and instrumentation talks. Science sessions were organized under themes that closely followed those in the US Decadal Survey. These themes stretched all the way from the first luminous objects in the Universe to our very own Solar System, while encompassing a diverse mix of black hole, galaxy cluster, quasar, stellar and exoplanetary science. Furthermore, new science opportunities were discussed, including the extreme physics of optical/near-infrared transients and resolved imaging of the surface of Kuiper Belt Objects.

The flexibility of TMT observing modes in terms of seeing-limited and full MCAO-assisted observations coupled to fast response times is expected to be a powerful enabler of new discoveries in an era of large imaging surveys. New technologies for future instruments were described, and the unique science potential of TMT for observing Jupiter-like exoplanets in reflected light, young Jovians in distant star-forming regions and characterizing exoplanetary atmospheres was re-affirmed. Although the emphasis of the workshop was on the science and technical concepts for the second generation of TMT instruments, the three first light instruments (IRIS, a diffraction limited NIR imager and integral field

Ground-based and Airborne Instrumentation for Astronomy IV, edited by lan S. McLean, Suzanne K. Ramsay, Hideki Takami, Proc. of SPIE Vol. 8446, 84461F · @ 2012 SPIE · CCC code: 0277-786X/12/\$18 · doi: 10.1117/12.930500 
spectrograph; WFOS, a wide field optical spectrograph; and IRMS, a near-infrared, multi-slit spectrograph) received their fair share of attention with detailed updates from the teams on their current progress. It was interesting to note that even though these instruments were selected at the end of 2006, their selection has withstood the test of time by providing the right balance of workhorse capabilities between imaging versus spectroscopy, optical versus infrared wavelengths and seeing-limited versus AO-assisted observing, and excellent synergy with other facilities such as ALMA and JWST. In terms of desired future capabilities, high spectral resolution spectroscopy and high contrast imaging ranked high amongst the workshop participants.

\subsection{Cost Review}

TMT held a project-wide, multi-day cost review in January 2011 by an external, non-advocate panel of international experts involved in a variety of large scientific projects. In preparation for this review, cost and schedule estimates for all observatory subsystems (including the instruments) were updated based on the latest information. All TMT cost and estimates are developed using a well-defined, detailed, bottom-up methodology. Each element of the TMT WorkBreakdown-Structure (WBS) has an associated "cost sheet" that captures estimates for labor, non-labor and travel costs as well as the bases for these estimates. Contingency is also estimated on each cost sheet using a stringent matrix that takes into account technical, cost and schedule risks. The TMT Cost book contains nearly 1500 pages with more than 650 cost sheets. Cost estimates are tied to a detailed Integrated Project Schedule with more than four thousand activities.

Detailed cost and schedule estimates were thus developed for the three early-light instruments, NFIRAOS (the first light MCAO system), LGSF (the Laser Guide Star Facility), the NSCU (NFIRAOS Science Calibration Unit) as well as overall instrument management and systems engineering activities. These estimates are regularly updated and further developed as designs progress. They are proving to be essential in informing the TMT Board and Science Advisory Committee of the financial impact of design trade-offs as they arise.

\subsection{Forming Global Partnerships}

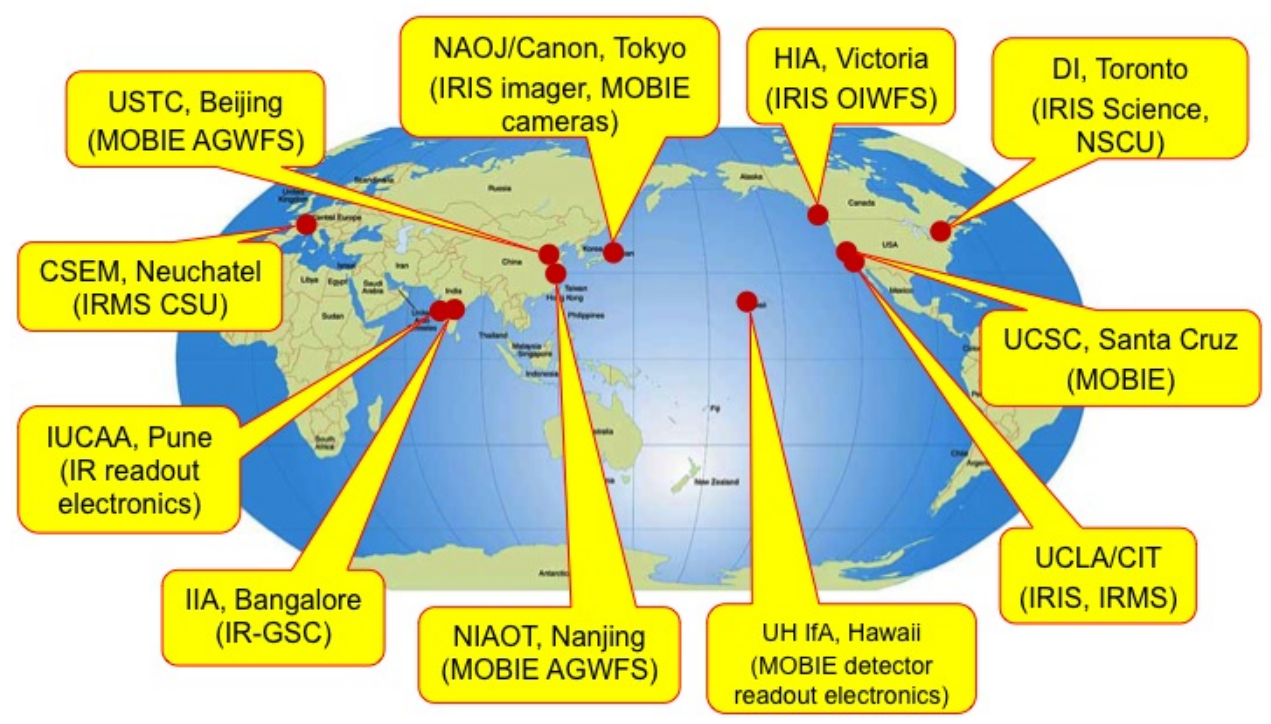

Figure 1. TMT science instrument contributions from around the world. MOBIE is the latest design for WFOS as discussed in Section 3.1.

Instruments for extremely large telescopes represent very sizable investments of resources, and each instrument will have to be built by a consortium of institutions. This is a continuation of the evolution seen in building ever more ambitious instruments for existing 4-m and 8-m class telescopes. All TMT partners have a strong interest in participating in instrument projects, and this interest is driven primarily by science interests within their respective communities. Partners are overcoming the substantial differences in cultures, the large geographical distances and different 
development models. There is a broad range of facilities and capabilities across all partner countries. Significant efforts have been (and will continue to be) made to fully realize the exciting potential found within the TMT partnership. Figure 1 shows the world map of TMT science instrument contributions as it currently stands. The overarching goal here is to form new instrument collaborations that make sense scientifically and technically while satisfying partner aspirations. Instrument partnerships will likely evolve as instrument designs progress further.

\section{PROGRESS ON FIRST-LIGHT INSTRUMENT DESIGNS}

The TMT first-light instruments were selected at the end of 2006 by the TMT SAC. This downselect was very successful because even though it was primarily science-driven, it also paid attention to technical readiness, costs and schedules. It was also based on extensive information from instrument feasibility studies. The selection of the first-light instruments responded to a natural balance between different fundamental observing modes: seeing-limited versus AO-assisted, visible versus infrared and imaging versus spectroscopy, and they also provided workhorse capabilities and excellent synergy with existing and future facilities. Given all of these considerations, it was not surprising that this instrument selection was re-confirmed in March 2011 by SAC at a meeting on the heels of the Science and Instrumentation workshop. Information on the planned suite of first-light and future TMT instruments can be found in the Instrumentation and Performance Handbook ${ }^{2}$.

\subsection{Wide-Field Optical Spectrograph (WFOS)}

The fundamental science parameters of a spectrograph are field of view, total slit length, spectral resolution and simultaneous wavelength coverage. There is a tension between these parameters depending on the types of spectroscopy programs that will be carried out. "Survey spectroscopy" (e.g., redshift determination) needs to achieve high multiplexing factors by maximizing both field of view and total slit length. "Diagnostic spectroscopy" (e.g., chemical abundances through line ratios) requires wide wavelength coverage to simultaneously measure multiple lines and control systematics and relatively high spectral resolution to separate out line blends. Reconciling both types of spectroscopy within a single instrument is quite challenging. The latest design for TMT's Wide-Field Optical Spectrograph (WFOS) is the Multi-Object Broad-band Imaging Echellelette (MOBIE) spectrograph, and it provides an elegant solution to that challenge. It can trade multiplexing for expanded wavelength coverage in its higher dispersion with the proper selection of a combination of grating, cross-dispersing prism and order-sorting filter. MOBIE's wavelength range is 0.31-1.1 $\mu \mathrm{m}$ with throughput higher than $40 \%$ at all wavelengths. The blue sensitivity of MOBIE was deemed to be of high science importance in probing important metal transitions in the intergalactic medium at all redshifts and in observations of extremely metal-poor stars. With its total field of view of $40 \mathrm{arcmin}^{2}$, and its total slit length of 576 arcseconds, MOBIE can observe as many as 200 objects simultaneously at low spectral resolution. The current design offers spectral resolutions of $\mathrm{R}=1000,5000$ and 8000 for a $0^{\prime \prime} .75$ slit width. Achieving higher resolutions will be possible with narrower slits especially for point source observations. Figure 2 shows a schematic view of the current design. The cylindrical instrument assembly is four meters in diameter.

MOBIE is a collaboration between the University of California at Santa Cruz (UCSC), the California Institute of Technology (CIT), the Nanjing Institute of Astronomical Optics and Technology (NIAOT), the University of Science and Technology of China (USTC), the National Astronomical Observatory of Japan (NAOJ), and the University of Hawaii (UH). The principal investigator is Rebecca Bernstein (UCSC), the project manager is Bruce Bigelow (UCSC), and the project scientist is Charles Steidel (CIT). NIAOT/USTC are responsible for the design of the acquisition, guiding and wavefront-sensing camera (AGWFS). NAOJ is working on the opto-mechanics of the blue and red spectrograph cameras, and UH is developing the instrument software requirements and architecture and designing the detector readout electronics. Many commercial vendors are also involved in various subsystem studies.

MOBIE is currently halfway through its Conceptual Design Phase (CDP). For a number of programmatic reasons, the MOBIE CDP was organized as two separate stages. Stage 1 was completed in January 2011. It provided an optical design for the spectrograph including initial sensitivity and stray light analyses, mechanical and opto-mechanical designs for the slit masks, the guider and wavefront sensor, the collimator mirror, the prisms and motion stages, the science detector systems and instrument structures. Stage 1 also delivered a new draft science case and Operational Concepts Definition Document. The last six months of Stage 1 were devoted to an extensive bottom-up cost and schedule estimate effort in preparation for the TMT January 2011 Cost Review. The Cost Review was then followed by a valueengineering study that looked at various trades between requirements (field of view, spectral resolution, and wavelength 
coverage) and cost and provided a selection of descope/upscope options. Stage 2 was initiated in March 2012. Stage 2 activities include finalizing optical and mechanical conceptual designs for all subsystems, defining electronics and software requirements/interfaces, and updating cost and schedule estimates. Stage 2 science activities will produce revised science cases and operational concepts, a data simulator and exposure time calculator and requirements for data reduction pipelines for imaging and spectroscopic modes. Single point diamond turning of aspheric surfaces on a large (D 350mm) piece of $\mathrm{CaF}_{2}$ will also be prototyped in this stage.

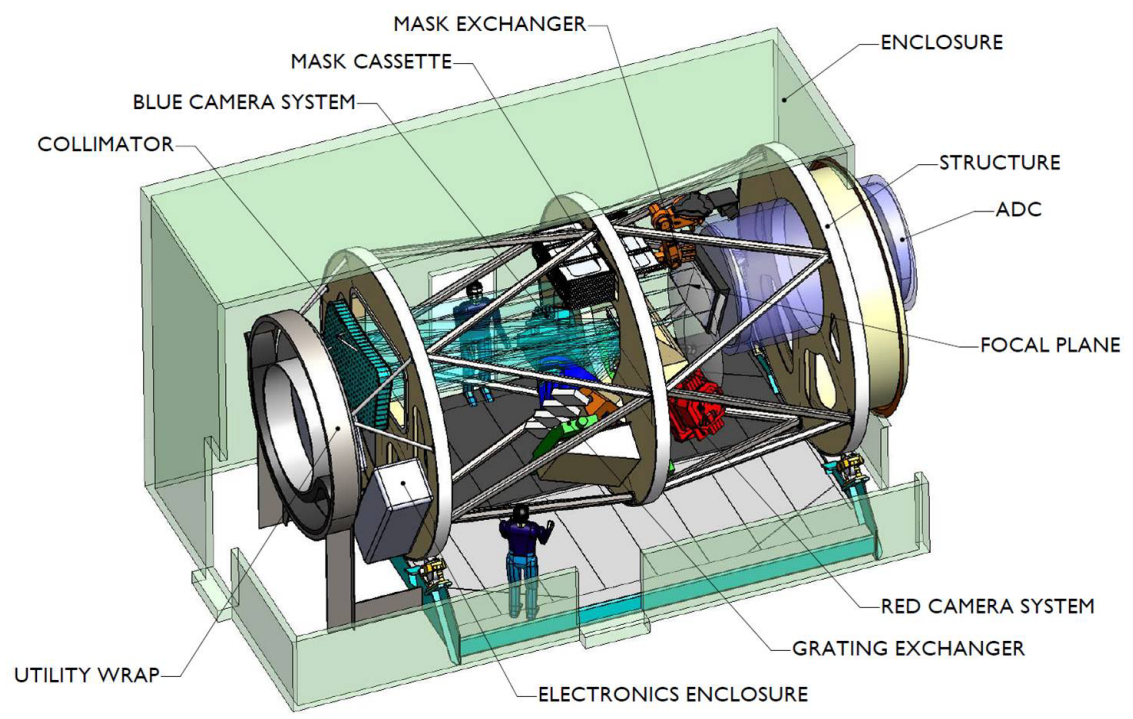

Figure 2. Schematic view of WFOS-MOBIE (Courtesy: Bruce Bigelow, UCO/Lick Observatory)

MOBIE is described in more details elsewhere ${ }^{3}$ in these proceedings.

\subsection{InfraRed Imaging Spectrograph (IRIS)}

IRIS is an integral field spectrometer and imager designed to work with the NFIRAOS Multi-Conjugate Adaptive Optics (AO) System over the 0.84-2.4 $\mu \mathrm{m}$ wavelength range. It will thus provide diffraction-limited imaging for precision photometry and astrometry over a sizable, uniform field $\left(16^{\prime \prime} .4\right.$ by $\left.16^{\prime \prime} .4\right)$ and integral field spectroscopy with a spectral resolution of $\mathrm{R}=4000$ (with $\mathrm{R}=8000$ as an option currently under study) at different spatial scales $\left(0^{\prime \prime} .004,0^{\prime \prime} .010\right.$, $0^{\prime \prime} .025$ and $0^{\prime \prime} .050$ per pixel). IRIS is optimized to exploit the full potential of a $30-\mathrm{m}$ telescope for precision imaging and spectroscopy at high spatial resolution and very faint limits, capitalizing on the $\mathrm{D}^{4}$ gain for point-like sources in the diffraction-limited regime. To achieve these goals, IRIS includes a set of three near-infrared wavefront sensors that can be precisely positioned and locked onto AO-sharpened natural guide stars to control tip/tilt, focus and tilt anisoplanatism for NFIRAOS (blind modes in a Laser Guide Star system). The natural guide stars can be selected over a 2-arcminute field, providing better than 50 percent sky coverage with less than 2 milliarcseconds of jitter, even at the galactic poles. Astrometric accuracies of better than $50 \mu$ as in 100-second MCAO-corrected exposures will be achievable over the entire field of the imager. The imager will deliver images with total wavefront errors of less than $30 \mathrm{~nm}$.

An innovative "hybrid" design is used by the IRIS spectrometer to achieve integral field spectroscopy with both the highest possible image quality (using a lenslet array) and the highest possible sensitivity (using an image slicer). The lenslet integral field system will deliver spectra of objects with either 4 mas or 10 mas spatial sampling and a 5 percent spectral band pass; the image slicer spectrometer will deliver spatial sampling of either 25 mas or 50 mas over areas up to $2^{\prime \prime} .2$ by $4^{\prime \prime} .4$ in size, covering full near-infrared bands. Higher spectral resolutions (up to $R=10,000$ ) are achievable with more limited wavelength coverage. Only the image dissection technique changes in the spectrometer between the 
two hybrid modes: most of the foreoptics camera optics, the gratings and the detectors are common, significantly reducing cost and risk.

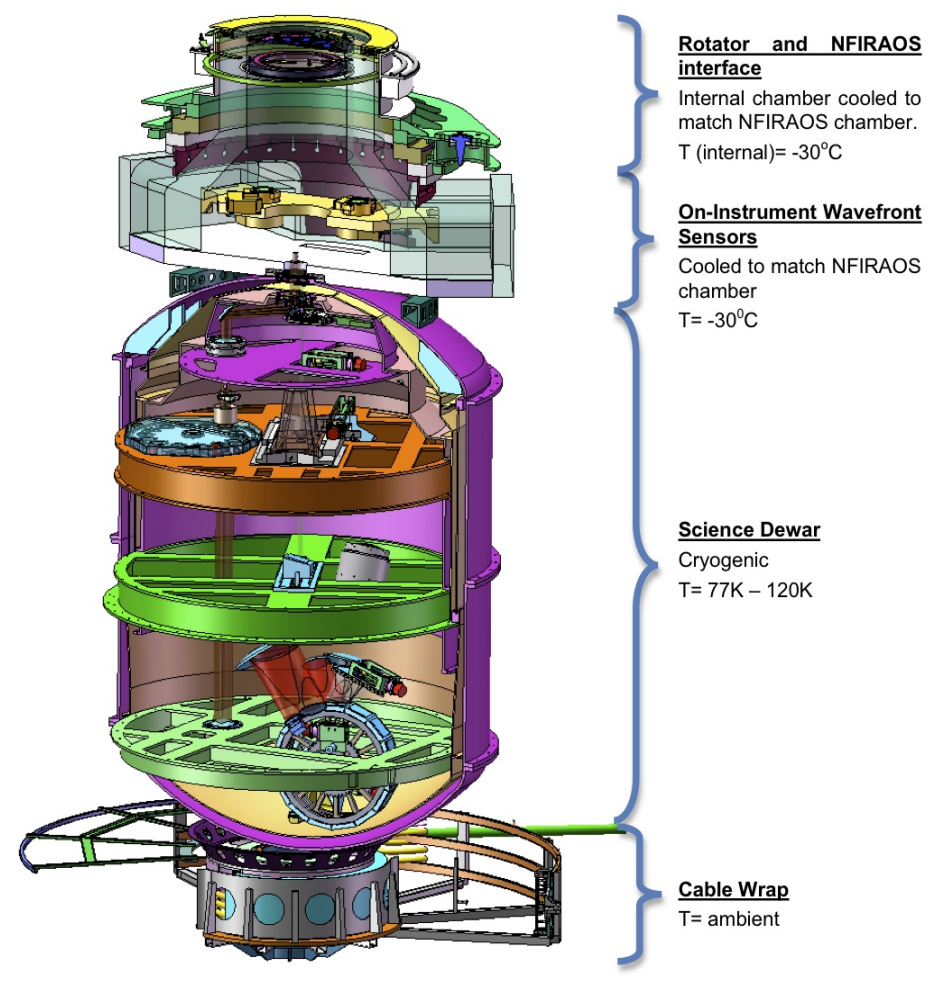

Figure 3. This cut-away of the IRIS instrument shows the major instrument sub-assemblies. Only the vacuum-sealed science dewar is maintained at cryogenic temperatures. The remaining modules are cooled to $-30^{\circ} \mathrm{C}$, the NFIRAOS enclosure temperature, or held at ambient. The science dewar contains the IRIS imager and IFS channels. IRIS is mounted on the bottom port of NFIRAOS (Source: IRIS Conceptual Design Study Report)

The following organizations are involved in the IRIS science and technical teams: California Institute of Technology, University of California (Los Angeles, Irvine, Santa Cruz, Berkeley), Herzberg Institute of Astrophysics, University of Toronto (UofT) and National Astronomical Observatory of Japan. The co-PIs are James Larkin (UCLA) and Anna Moore (CIT). The project scientist is Shelley Wright (UofT). The IRIS design greatly benefits from the legacy of Keck/OSIRIS, Keck/MOSFIRE and Gemini/GPI instruments. The IRIS team successfully passed their Conceptual Design Review in December 2011. Figure 3 shows an exploded view of the full IRIS assembly. In addition to the instrument design, this conceptual design phase produced new sets of science cases and operational concepts as well as a full data simulator capable of producing realistic datacubes for a wide range of observations to characterize the expected performance of the instrument (see Table 1).

The IRIS conceptual design study identified three critical subsystems that should be prototyped as the instrument effort moves beyond conceptual design, and these prototypes are being built and tested in the lab. This prototyping phase will be completed in early 2013. The three prototypes are 1) the on-instrument wavefront sensor probe arms (Figure 4), 2) the spectrograph grating turret and 3) the Spinel glass to be used in the atmospheric dispersion correctors. The root-meansquare blind positioning accuracy of the OIWFS probes in the f/15 NFIRAOS focal plane is 2 milli-arcseconds. This corresponds to 4 microns at the TMT plate scale of $0.458 \mathrm{arcsec} / \mathrm{mm}$. The full patrol field has a diameter of $26 \mathrm{~cm}$. The probe arms for the sensors use a Theta-R configuration to achieve the desired positioning accuracy; it is also easier to manage collision avoidance with such a configuration. The probe arms are designed to work at a temperature of $-30^{\circ} \mathrm{C}$. A prototype is needed to test robust operation and mechanical accuracy of the probe arm under low temperature conditions. The spectrograph grating turret will have up to 15 positions and require micron level angular repeatability. 
Several turret concepts have been put forward based on more primitive mechanisms from the MOSFIRE and GPI instruments. Demonstrating a detent system that will hold micron tolerances even without power at the expected

Table 1. IRIS Point Source Sensitivities. Imaging point source sensitivities use a $2 \lambda / D$ aperture size to achieve a signal-to-noise of 100 in a 5-hour integration. Spectroscopy point source sensitivities are for a scale of 4 milliarcseconds and use a $2 \lambda / \mathrm{D}$ aperture size to achieve a signal-to-noise per spectral channel of 10 between $\mathrm{OH}$ sky emission lines (Source: IRIS Operational Concepts Definition Document).

\begin{tabular}{|c|c|c|c|}
\hline \multicolumn{5}{|c|}{ Imaging } \\
\hline Filter & Exposure Time (s) & Number of Frames & Magnitude (AB) \\
\hline Y & 900 & 20 & 28.4 \\
\hline J & 900 & 20 & 27.8 \\
\hline H & 900 & 20 & 27.3 \\
\hline K & 300 & 60 & 26.9 \\
\hline \multicolumn{2}{|c|}{ Spectroscopy } & Magnitude (AB) \\
\hline Filter & Exposure time (seconds) & Number of Frames & 26.20 \\
\hline Y & $900 \quad 20$ & 26.42 \\
\hline J & 900 & 20 & 26.39 \\
\hline H & 900 & 60 & 25.13 \\
\hline K & 300 & & \\
\hline
\end{tabular}

operating temperature of $77 \mathrm{~K}$ is essential for moving forward with the existing optical design that utilizes multiple gratings. Finally, IRIS will have five atmospheric dispersion correctors: one ADC for each of the imager, IFS and three on-instrument wavefront sensors. These ADCs must deliver milliarcsecond-scale correction residuals. The current ADC design uses a pair of counter-rotating Amici prisms where pairs of glass materials have been optimized to match the
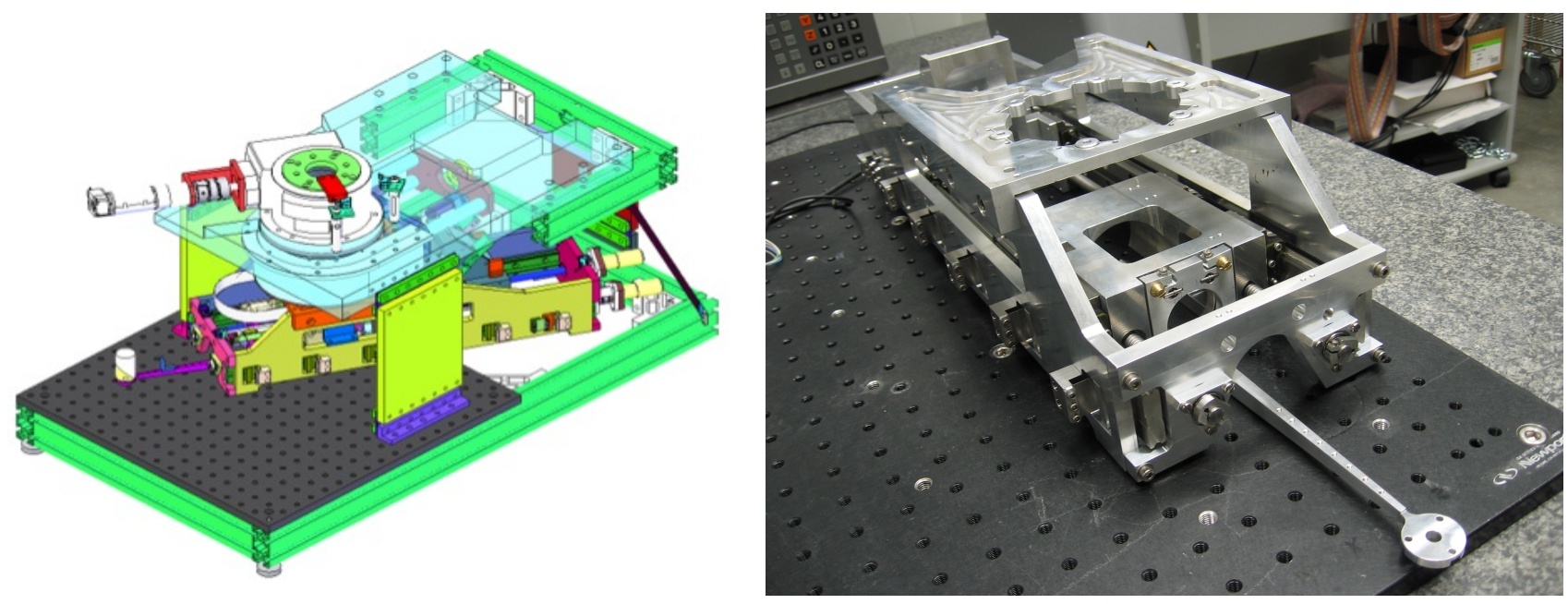

Figure 4. Mechanical prototype of the IRIS On-Instrument Wavefront Sensor probe arm (Courtesy: Vlad Reshetov, $N R C-H I A)$. The linear actuators have been assembled and tested in the cold room at $-30^{\circ} \mathrm{C}$ with no signs of seizure or uneven/increased torques. 
dispersion properties of the atmosphere on Mauna Kea. After looking at over 250 different glass combinations during the IRIS conceptual design phase, Spinel was shown to be the leading glass material. Spinel glass produces some of the best dispersion corrections in the near infrared, but its design purpose is as a bullet-proof glass. Since it is not typically grown for optical quality applications, it may have internal scattering issues that would prohibit its use. It is therefore important to ascertain its availability and characterize its optical performance at room and cryogenic temperatures.

More details on IRIS are given at http://irlab.astro.ucla.edu/iris.

\subsection{InfraRed Multi-slit Spectrograph (IRMS)}

IRMS is a clone of the Keck MOSFIRE instrument now undergoing on-sky commissioning. MOSFIRE is a multi-slit instrument designed for the $\mathrm{f} / 15$ Cassegrain focus on the Keck 1 telescope. This is the same $\mathrm{f} / \mathrm{ratio}$ as for the TMT Nasmyth focus. Without changes to the MOSFIRE design, it would naturally take in the entirety of the NFIRAOS field of regard of $2^{\prime}$ in diameter and cover the wavelength range $0.95 \mu \mathrm{m}$ to $2.45 \mu \mathrm{m}$ with spectral resolution of up to $\mathrm{R}=4660$ for a $0^{\prime \prime} .16$ slit. MOSFIRE uses a cryogenic slitmask unit (CSU) that was originally developed as a backup for the JWST NIRSPEC instrument. The spatial pixel scale (50 mas) on TMT and the length of individual slitlets in IRMS (made by masking bars in MOSFIRE) is reasonably well matched to the sampling scale requested in the TMT Science Requirements Document. For multiplexing, the individual bars can be configured in up to 46 slitlets over the entire NFIRAOS field; in practice, some of the slitlets would be made into contiguous slits of lengths that are multiples of $2^{\prime \prime} .4$. The width of slits and their placement within the field are remotely configurable in real time. IRMS on TMT will also offer additional capabilities. It can be configured as a long slit spectrograph or as an imager that would cover the entire NFIRAOS field of regard, albeit with spatial sampling of only 33 mas to 60 mas (roughly two to four times larger than the diffraction limit at $2 \mu \mathrm{m}$ ). It can also be used in a seeing limited mode (for either imaging or spectroscopy), if desired, by flattening the deformable mirrors in NFIRAOS and turning off the AO correction.

Two studies are underway to assess IRMS on TMT. The first study led by Bahram Mobasher at UC Riverside focuses on science performance. The primary goals of this study are to develop existing and new science cases to a greater level of details, to identify and defined new scientific requirements for IRMS, to define observing scenarios that include target selection procedures, daytime and nighttime calibrations and on-sky acquisition strategies and to develop a full data simulator. Initial results from this study (see Figure 5) already show that TMT/IRMS will reach at least two magnitudes fainter in the $\mathrm{J}$ and $\mathrm{K}$ bands than Keck/MOSFIRE.

Although IRMS is intended to be a clone of MOSFIRE as much as possible, some changes to the MOSFIRE designs are unavoidable. An initial investigation to ascertain the feasibility of feeding a MOSFIRE clone with NFIRAOS was carried out back in 2007, and its basic conclusions were that 1) IRMS should work well behind NFIRAOS, 2) the first field lens would require a different prescription and be moved slightly, and 3) all the remaining optics (including the CSU and mechanical systems) could remain the same. Subsequently, the NFIRAOS optical design was changed from a 2-OAP to a 4-OAP design to minimize distortion. The reduced distortion is essential to IRMS, but unfortunately, the focal surface became much more curved, with the curvature in the opposite sense to that of Keck. It was also recognized that new designs might be required for the interface to NFIRAOS, the rotator, the thermal baffle in the front of the instrument and the guider probe system. Given all of these uncertainties, a study was initiated at CIT in collaboration with the MOSFIRE team to identify and design the changes needed to MOSFIRE to meet the IRMS requirements on TMT. This study is to be completed by December 2012, but results obtained so far show no obstacles. The MOSFIRE optical design requires minimal changes that do not affect the mechanical envelopes of subsystems such as the collimator, and MOSFIRE fits in the current mechanical and thermal interface of the NFIRAOS instrument port with no major front-end changes. Work is now focusing on the sky coverage and tip-tilt correction performance of the existing MOSFIRE wavefront sensor guide probe design. This is a single probe that has to patrol the upper or lower part of the field of view to avoid vignetting the middle part of the field where slitlets will be placed on science targets. Cost and schedule are of course also of prime importance, and the latest figures from the now-completed MOSFIRE project will prove to be a very reliable basis of estimate for the cost of IRMS on TMT. In some sense, the first instrument for TMT has already been built. 

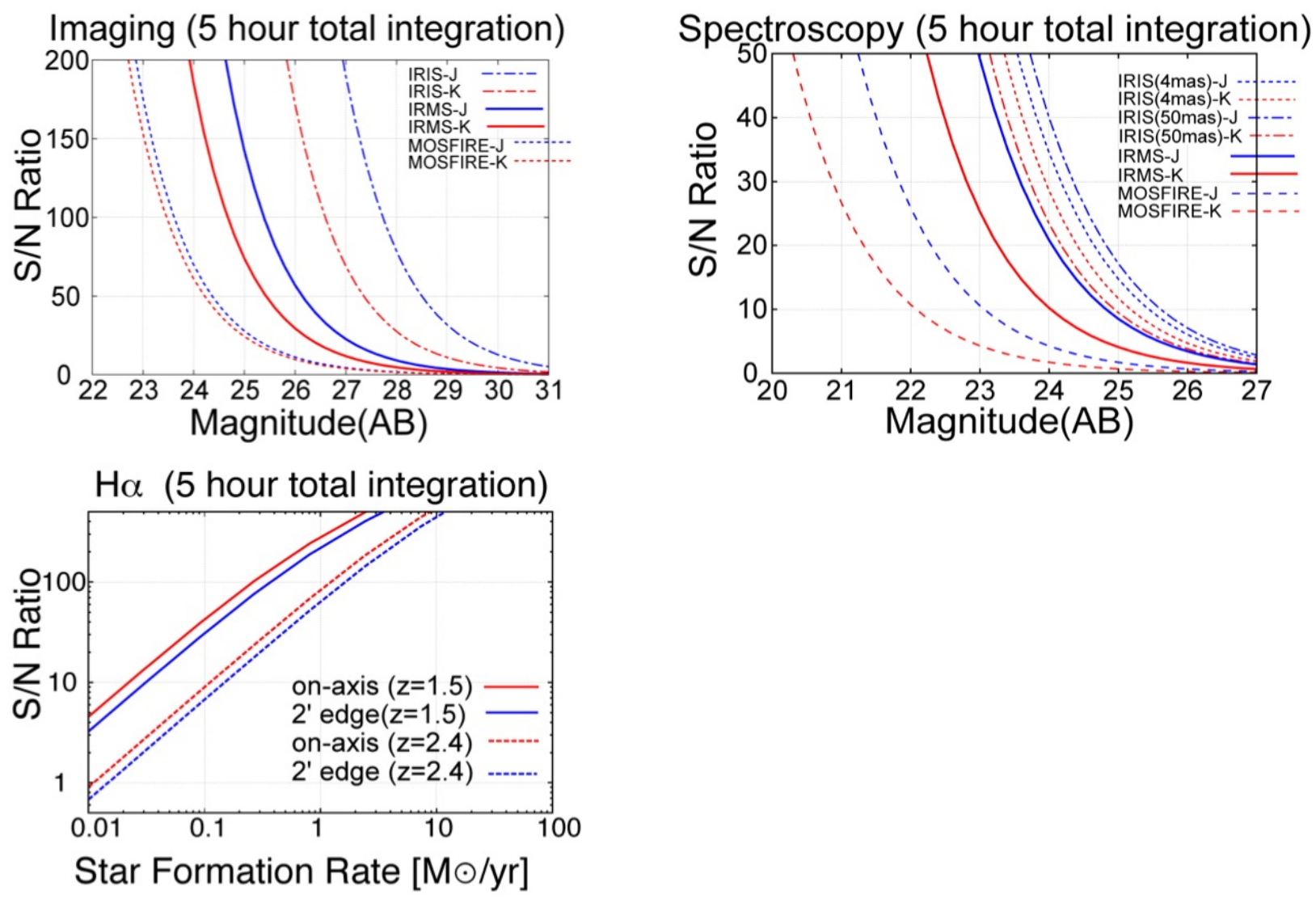

Figure 5. IRMS on TMT science performance versus Keck/MOSFIRE and TMT/IRIS in J, H and K for imaging, spectroscopy and H-alpha emission line (Courtesy: Chihiro Tokoku UC Riverside/U. Tokyo)

Details on MOSFIRE can be found at http://www.astro.ucla.edu/ irlab/mosfire/.

\section{NEW FUTURE INSTRUMENT CONCEPTS}

The first step in the development of new instrument concepts for TMT consists of community explorations (e.g., science and technical workshops, mini-studies, technology testbeds, etc. etc.). This is where new instrumentation ideas for TMT are born. Explorations are meant to inform the prioritization of desired TMT instrumentation capabilities by the TMT Scientific Advisory Committee. This prioritization must be primarily science-driven, but it must also factor in all available information on technical readiness, schedule, cost and the overall mix of commissioned and planned instrument at TMT. Given that both the scientific and technical landscapes can change significantly over time, such explorations must be conducted on a regular basis. While the first-light instruments were selected in 2006, it is important to emphasize that work on new concepts is proceeding on an ongoing basis within all TMT partner communities. Two concepts are briefly described here as examples and are described in greater details elsewhere in these proceedings.

\subsection{SEIT}

The direct imaging of exoplanets is a key capability for extremely large telescopes. The current work on high-contrast imagers such as Subaru/HiCIAO, Gemini/GPI and VLT/SPHERE will provide invaluable input into design efforts for ELT imagers. The performance of an exoplanet imager is primarily based on two parameters: the Inner Working Angle (IWA) and the contrast ratio. Different concepts explore different trade-offs between these two parameters depending on their science goals. A given planet type will be brighter at smaller IWAs, so the requirement on contrast ratio can be then relaxed. A team led by Taro Matsuo at the National Astronomical Observatory of Japan has been studying a concept 
called SEIT for "Second-Earth Imager for TMT". The SEIT concept is optimized for small IWAs $(\sim 1.5 \lambda / \mathrm{D}$ at $1 \mu \mathrm{m})$ with the specific science goal of imaging Earth-like planets in the habitable zone around K- and M-type stars.

SEIT is described in more details elsewhere ${ }^{4}$ in these proceedings.

\subsection{MICHI}

The original concept for a mid-infrared instrument on TMT was the Mid-InfraRed Echelle Spectrometer (MIRES) ${ }^{5}$. It brought diffraction-limited, high spatial resolution imaging and high-resolution spectroscopy in the thermal infrared (5 $\mu \mathrm{m}$ to $25 \mu \mathrm{m}$ ). Many of its science objectives required a high (greater than $4000 \mathrm{~m}$ ) and dry (less than $3 \mathrm{~mm}$ of water vapor) observatory site. Until an adaptive secondary becomes available, this concept would use a dedicated AO module (MIRAO) optimized for operation at those wavelengths. MIRES offered three parallel optical channels: a near-IR (Kband) acquisition camera with a $60^{\prime \prime}$ by $60^{\prime \prime}$ field of view, a mid-IR slit viewer that could double as a $4.5 \mu \mathrm{m}$ to $25 \mu \mathrm{m}$ science imager were the reflective slit be replaced by a mirror, and a mid-IR, cross-dispersed spectrograph capable of delivering spectra over the range $4.5 \mu \mathrm{m}$ to $25 \mu \mathrm{m}$ with an $\mathrm{R} \sim 100,000$. The MIRES concept was primarily driven by galactic science, and it lacked a low-spectral resolution capability for extragalactic science.

A new concept called MICHI for Mid-Infrared Camera, High-disperser, \& IFU spectrograph has been under study by a joint Japan-US team from Kanagawa University, Ibaraki University, the University of Hawaii and the University of Florida since 2008. MICHI adds key new capabilities: a much improved imager, a low-R IFU capability in the N-band (7-14 $\mu \mathrm{m})$, an internal cold chopper, and polarimetry. A high-contrast imaging option is also under consideration.

MICHI is described in more details elsewhere ${ }^{6}$ in these proceedings.

\section{OTHER INSTRUMENT-RELATED OBSERVATORY SUBSYSTEMS}

One of the goals of the TMT project is to integrate adaptive optics as an end-to-end part of its observatory design, and this goal places requirements on many observatory subsystems including instrument cooling. Minimizing vibration is understood to be a key requirement for any AO-assisted telescope. In fact, vibration has been (and continues to be) a limiting factor on the AO performance of instruments on existing telescopes, and a particularly bothersome source of vibration has been the instrument cryocoolers themselves. The ideal solution to this problem is to remove noisy mechanical components such as compressors from the immediate environment of the instruments. One example is the cooling architecture developed for the LINC-NIRVANA interferometric instrument at the $\mathrm{LBT}^{7}$. The cryostat is cooled by a flow of helium gas, and the gas cooler is placed far away on a different level in the telescope building. The cold helium is fed through long vacuum-isolated transfer lines to the instrument cryostat. A similar architecture is being explored for facility instrument cryogenic cooling system at TMT.

The planned suite of instruments for TMT would require a total cooling power of about $1.7 \mathrm{~kW}$ at $77 \mathrm{~K}$. Nasmythmounted instruments imply that transfer lines from the TMT utility room in the Summit Facility would have to go through the telescope azimuth wrap and individual instrument rotators. The total line length prior to the instrument rotator would typically be $\sim 125$ meters. Although different types of cryocoolers will be studied including Stirling and pulse-tube coolers, special attention will be paid to gas turbine expanders (also known as Reverse-Brayton or TurboBrayton). Like Joule-Thomson coolers, they use adiabatic expansion that is isentropically reversible to cool the gas as work is done on the turbine. High RPM gas bearings result in very low vibration. Large expanders are commonly found in air separation or helium liquefaction facilities. Miniature sizes have been built for space missions but at high cost due to complexity of miniaturization and small production numbers. No medium-sized turbo-expanders are currently available commercially. One option for TMT might be to use a single large turbine located on one of the Nasmyth platforms. Helium gas would then be sent from the utility room to the expander at ambient temperature, and vacuumisolated transfer lines would take cold helium gas from the expander to all the instruments. One potential problem with this option is the stress on the transfer lines due to the small radius of curvature of instrument rotators. Another problem would be reliability. Downtime of a single expander would interrupt cooling to all instruments. An interesting alternative is to consider mounting a small turbine expander on each instrument. Such a concept is illustrated in Figure 6. Helium gas is run at ambient temperature all the way from the utility room to the instrument without the need for vacuumisolated transfer lines. TMT is working with the cryogenic cooling group at the Technical Institute of Physics and Chemistry (TIPC) in Beijing to develop "mid-size" turbine expanders ( 300W @ 77K). The TIPC group has extensive experience with larger expanders, and prototypes for smaller ones are already being built and tested under laboratory 
conditions. The successful development of smaller expanders has the potential to lead to a paradigm shift in the way cryogenic cooling is delivered to vibration-sensitive, AO-assisted instruments. Needless to say, this development work will be carefully assessed in terms of its technical merit, cost and schedule against a benchmark case using a variety of "conventional" cryocoolers.

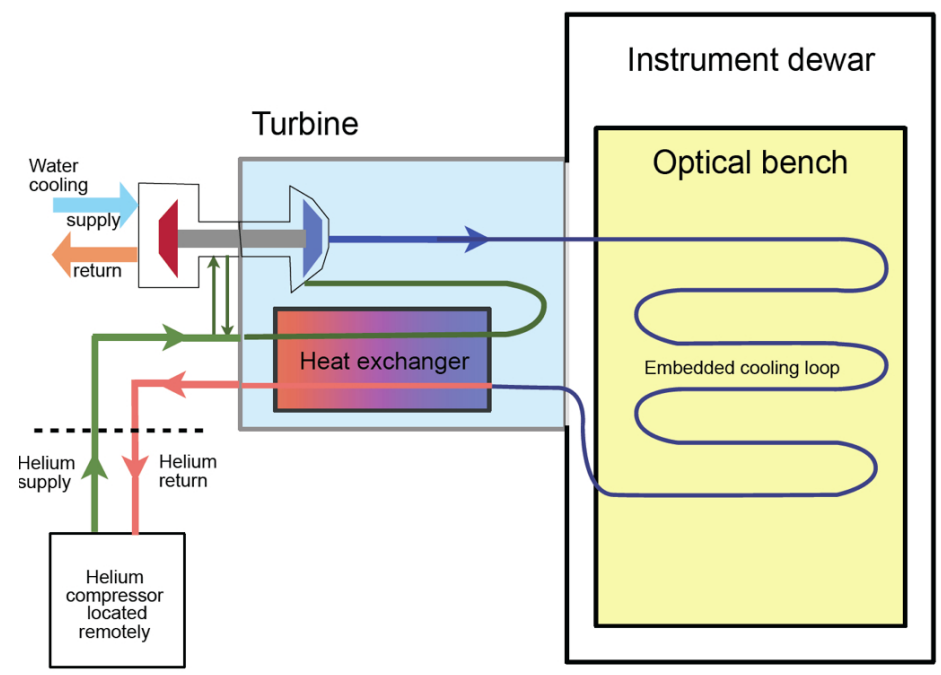

Figure 6. Schematic diagram of an instrument cooling system based on an on-board turbine expander (Courtesy: Hugh Thompson, TMT and Liqiang Liu, TIPC).

\section{ACKNOWLEDGEMENTS}

The TMT Project gratefully acknowledges the support of the TMT collaborating institutions. They are the Association of Canadian Universities for Research in Astronomy (ACURA), the California Institute of Technology, the University of California, the National Astronomical Observatory of Japan, the National Astronomical Observatories of China and their consortium partners, and the Department of Science and Technology of India and their supported institutes. This work was supported as well by the Gordon and Betty Moore Foundation, the Canada Foundation for Innovation, the Ontario Ministry of Research and Innovation, the National Research Council of Canada, the Natural Sciences and Engineering Research Council of Canada, the British Columbia Knowledge Development Fund, the Association of Universities for Research in Astronomy (AURA) and the U.S. National Science Foundation.

\section{REFERENCES}

[1] Ellerbroek, B. L. et al., "TMT adaptive optics program status,” Proc. SPIE 8447-55 (2012)

[2] TMT Instrumentation and Performance Handbook (www.tmt.org/sites/default/files/TMT-Instrumentation-andPerformance-Handbook.pdf)

[3] Bernstein, R. A., \& Bigelow, B. C., "Conceptual Design Status of the MOBIE Imaging Spectrograph for the TMT," Proc. SPIE 8446-51 (2012)

[4] Matsuo, T. et al., "Second-Earth Imager for TMT (SEIT)," Proc. SPIE 8446-56 (2012).

[5] Tokunaga, A. T. et al., "Design tradeoffs for a high spectral resolution mid-infrared echelle spectrograph on the Thirty-Meter Telescope," Proc. SPIE, 6269, 126 (2006)

[6] Packham, C. C. et al., "MICHI: A mid-IR instrument concept for the TMT," Proc. SPIE 8446-287 (2012)

[7] Laun, W., Baumeister, H., and Bizenberger, P., "The LINC-NIRVANA IR cryostat," Proc. SPIE, 6269, p. 163 (2006) 